\title{
OPEN The incidence of severe urinary tract infection increases after hip fracture in the elderly: a nationwide cohort study
}

\author{
Yi-Ching Lin ${ }^{1,6}$, Ya-Chu Hsu ${ }^{1,6}$, Wen-Tien Wu $\mathbf{u}^{1,2,3}$, Ru-Ping Lee ${ }^{3}$, Jen-Hung Wang ${ }^{4}$, \\ Hao-Wen Chen ${ }^{2}$, Ing-Ho Chen ${ }^{1,2}$, Tzai-Chiu Yu ${ }^{1,2}$, Cheng-Huan Peng ${ }^{2}$, Kuan-Lin Liu ${ }^{1,2}$, \\ Chung-Yi Hsu ${ }^{5}$ \& Kuang-Ting Yeh ${ }^{1,2 \bowtie}$
}

\begin{abstract}
Although urinary tract infection (UTI) is a common perioperative complication among elderly patients with hip fracture, its incidence and effects are often underestimated. This study investigated the effects of severe UTI (S-UTI) on elderly patients with hip fracture and the risk factors for this condition. In this retrospective nationwide cohort study, we searched Taiwan's National Health Insurance Research Database from 2000 to 2012 for data on patients aged $\geq 50$ years with hip fracture who underwent open reduction and internal fixation or hemiarthroplasty for comparison with healthy controls (i.e. individuals without hip fracture). The study and comparison cohorts were matched for age, sex, and index year at a 1:4 ratio. The incidence and hazard ratios of age, sex, and multiple comorbidities associated with S-UTI were calculated using Cox proportional hazard regression models. Among the 5774 and 23,096 patients in the study and comparison cohorts, the overall incidence of S-UTI per 100 person-years was 8.5 and 5.3 , respectively. The risk of S-UTI was cumulative over time and higher in the study cohort than in the comparison cohort, particularly in those who were older, were female, or had comorbidities of cerebrovascular accident or chronic renal failure.
\end{abstract}

As a society ages, the prevalence of osteoporotic fractures, such as hip fracture, increases. Hip fracture can considerably impair late-life function and substantially increase the medical burden. Prompt surgery and aggressive rehabilitation may increase the survival rate and improve the overall functioning of patients with hip fracture. The complication rates of treatment on admission range from 7 to $40 \%^{1,2}$. Immediate full weight-bearing mobilisation is targeted to prevent additional harm, such as pressure sores or pneumonia. However, pre-existing comorbidities and unsteady gait often make mobilisation difficult ${ }^{3}$.

A prospective study reported that 1 year after hip fracture, more than $20 \%$ of patients who were previously healthy required long-term care, and approximately $80 \%$ of them relied on a walking aid to perform daily life activities $^{4}$. Even with timely surgery and aggressive management through rehabilitation, hip fracture is associated with a high mortality rate. This mortality rate can mostly be attributed to the high prevalence of prefracture comorbidities and the high rate of postoperative complications, such as surgical wound infection, pneumonia, bleeding, ileus, delirium, and urinary tract infection (UTI $)^{5,6}$, which is a highly common postoperative complication in numerous surgical fields ${ }^{7}$. The rate of UTI after surgery for hip fracture may be underestimated and associated with inferior functional outcomes. ${ }^{8}$ UTI does not seem to affect the rate of perioperative wound infection or in-hospital mortality. However, severe UTI (S-UTI) often requires further intervention. S-UTI is defined as symptomatic UTI requiring hospitalisation to prevent severe adverse sequelae, including death. Studies on UTI during and after recovery from hip fracture have seldom been performed. This nationwide population-based cohort study investigated the incidence of and risk factors for S-UTI after hip fracture surgery. The present findings can serve as a reference for the provision of special care to reduce the risk of postoperative S-UTI, thereby improving the quality of life of affected patients and reducing medical expenditure.

\footnotetext{
${ }^{1}$ School of Medicine, Tzu Chi University, Hualien 97004, Taiwan. ${ }^{2}$ Department of Orthopedics, Hualien Tzu Chi Hospital, Buddhist Tzu Chi Medical Foundation, No. 707, Sec. 3, Zhongyang Rd., Hualien 97002, Taiwan. ${ }^{3}$ Institute of Medical Sciences, Tzu Chi University, Hualien 97004, Taiwan. ${ }^{4}$ Department of Medical Research, Hualien Tzu Chi Hospital, Buddhist Tzu Chi Medical Foundation, Hualien 97002, Taiwan. ${ }^{5}$ Graduate Institute of Clinical Medical Science, China Medical University, Taichung 406040, Taiwan. ${ }^{6}$ These authors contributed equally: Yi-Ching Lin and Ya-Chu Hsu. ${ }^{\circledR}$ email: micrograft@tzuchi.com.tw
} 


\begin{tabular}{|c|c|c|c|c|c|}
\hline & \multicolumn{4}{|l|}{ Hip fx } & \multirow[b]{3}{*}{ Standardized mean difference } \\
\hline & \multicolumn{2}{|c|}{ No $(n=23,096)$} & \multicolumn{2}{|c|}{\begin{tabular}{|l|}
$\begin{array}{l}\text { Yes } \\
(\mathrm{n}=5774)\end{array}$ \\
\end{tabular}} & \\
\hline & $\mathbf{n}$ & $\%$ & $\mathbf{n}$ & $\%$ & \\
\hline \multicolumn{6}{|l|}{ Surgery type } \\
\hline ORIF & & & 3469 & 60.1 & \\
\hline Hemiarthroplasty & & & 2305 & 39.9 & \\
\hline Sex & & & & & 0.05 \\
\hline Female & 12,514 & 54.2 & 3182 & 55.1 & \\
\hline Male & 10,582 & 45.8 & 2592 & 44.9 & \\
\hline Age (years) & & & & & 0.02 \\
\hline $50-59$ & 1900 & 8.2 & 472 & 8.2 & \\
\hline $60-69$ & 3550 & 15.4 & 869 & 15.1 & \\
\hline $70-79$ & 8540 & 37 & 2165 & 37.5 & \\
\hline $80+$ & 9106 & 39.4 & 2268 & 39.3 & \\
\hline Mean (SD) & 76.3 & 9.94 & 76.2 & 9.84 & \\
\hline \multicolumn{6}{|l|}{ Comorbidity } \\
\hline HTN & 16,942 & 73.4 & 4205 & 72.8 & 0.05 \\
\hline $\mathrm{DM}$ & 9148 & 39.6 & 2281 & 39.5 & 0.02 \\
\hline Hyperlipidemia & 7245 & 31.4 & 1802 & 31.2 & 0.01 \\
\hline CRF & 457 & 2 & 113 & 2 & 0.01 \\
\hline Depression & 2710 & 11.7 & 712 & 12.3 & 0.02 \\
\hline CAD & 10,803 & 46.8 & 2678 & 46.4 & 0.05 \\
\hline CVA & 8792 & 38.1 & 2193 & 38 & 0.01 \\
\hline Mean of follow-up period of S-UTI (Mean/SD) (years) & 4.14 & 3.19 & 3.74 & 3.19 & 0.09 \\
\hline
\end{tabular}

Table 1. Baseline characteristics in study cohorts with hip fx undergoing surgery and without hip fx. A standardized mean difference $\leq 0.10$ indicates a negligible difference between the two cohorts. $C A D$ coronary artery disease, $C R F$ chronic renal failure, CVA cerebrovascular accident, $D M$ diabetes mellitus, $F x$ fracture, HTN hypertension, ORIF open reduction and internal fixation, S-UTI severe urinary tract infection.

\section{Results}

The study and comparison cohorts comprised 5774 and 23,096 patients, respectively. The study cohort was further divided by method of hip fracture surgery. The open reduction and internal fixation (ORIF) and hemiarthroplasty groups contained 3469 (60.1\%) and 2305 (39.9\%) patients, respectively. The comparison cohort comprised patients from the same database without hip fracture during the same period. Patients with hip fracture who did not undergo surgery were excluded because they comprised less than $1 \%$ of patients with hip fracture and surgery is the primary intervention for this type of injury. No significant between-cohort differences were observed in terms of age; sex; follow-up period; or comorbidities, including hypertension (HTN), diabetes mellitus (DM), hyperlipidaemia, depression, coronary artery disease (CAD), cardiovascular accident (CVA), and chronic renal failure (CRF; Table 1). In both cohorts, patients with any history of S-UTI, those with a confirmed diagnosis of hospital-acquired UTI from prolonged Foley catheter placement, and those receiving antibiotics more than $24 \mathrm{~h}$ postoperatively before and during the admission period for hip fracture surgery were excluded. The cumulative incidence of S-UTI was significantly higher in the ORIF and hemiarthroplasty groups than in the control group, according to a Kaplan-Meier analysis (log-rank test, $P<0.001$; Fig. 1). The overall incidence ratios of S-UTI for the control, ORIF, and hemiarthroplasty groups per 100 person-years were 5.3, 8.3, and 8.7, respectively (Table 2). The ORIF and hemiarthroplasty groups exhibited an increased risk of S-UTI compared with the control group, even after adjustment for sex; age; and comorbidities of HTN, DM, CAD, hyperlipidaemia, CRF, CVA, and depression (adjusted hazard ratios [aHRs] $=1.65$ and $1.55,95 \%$ confidence interval [CI] $1.55,1.77$ and 1.44, 1.68, respectively). No significant differences were noted between the ORIF and hemiarthroplasty groups $(\mathrm{aHR}=0.93$, 95\% CI 0.85, 1.03; Table 2). The increased incidence of S-UTI in the ORIF group was observed across all strata. The same phenomenon was noted in the hemiarthroplasty group across all strata, except for the stratification by CRF (Table 2). Comorbidity analysis revealed that CRF and CVA were significantly associated with S-UTI incidence $(P=0.0097$ and 0.0038 , respectively; Table 3$)$. The aHR of S-UTI in men was 0.77 times higher than that in women $(95 \%$ CI $0.74,0.81)$. Compared with that in patients aged 50-59 years, the S-UTI risk was 1.87, 3.42 , and 5.57 times higher in patients aged 60-69 years (95\% CI 1.58, 2.22), 70-79 years (95\% CI 2.93, 4.01), and $>80$ years ( $95 \%$ CI 4.76, 6.52; Table 3), respectively. HTN, DM, CAD, hyperlipidaemia, and depression were all significantly associated with S-UTI incidence $(P<0.05$; Table 3$)$. The aHR for S-UTI in the patients with CRF was 4.85 times higher than that in those without CRF (95\% CI 4.45, 5.29). In the patients with CVA, it was 1.56 times higher than that in those without CVA $(95 \%$ CI 1.48, 1.64; Table 3). The aHRs for S-UTI interacting with CRF and CVA were 5.05 (95\% CI 4.58, 5.56) and 1.85 (95\% CI 1.50, 1.68) in the control group, 7.77 (95\% CI 


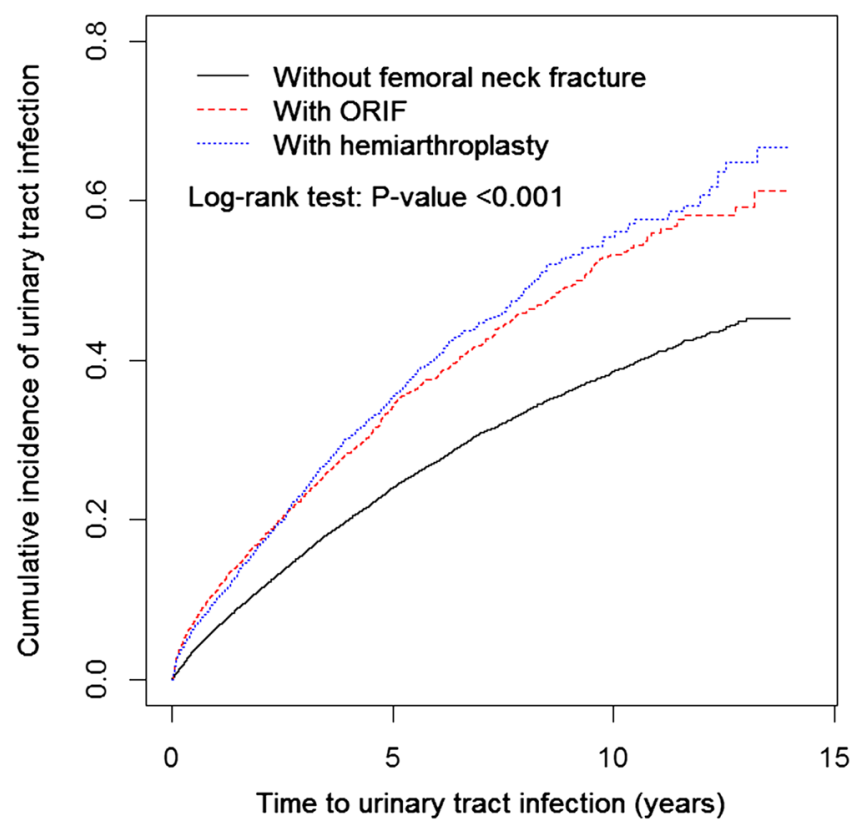

Figure 1. Cumulative incidences of urinary tract infection for ORIF, hemiarthroplasty and non-femoral neck fracture cohorts.

$6.06,9.97)$ and 2.55 (95\% CI 2.31, 2.81) in the ORIF group, and 5.85 (95\% CI 4.41, 7.76) and 2.32 (95\% CI 2.07, 2.60; Table 4) in the hemiarthroplasty group, respectively.

\section{Discussion}

This study discovered an increased risk of S-UTI after surgery for hip fracture compared with the risk in individuals without hip fracture. UTIs, which are among the most common bacterial infections in older patients with hip fracture $^{9}$, can be symptomatic or asymptomatic ${ }^{10}$. The present study examined the postoperative incidence of and risk factors for S-UTI to prevent its occurrence during recovery from hip fracture. The incidence of S-UTI was significantly higher in the study cohort than in the comparison cohort and increased over time. Older and female patients were even more likely to develop S-UTI. A 2005 study reported significant differences of $16 \%$ and $4 \%$ in UTI occurrence after hip fracture surgery between patients older and younger than 65 years, respectively ${ }^{11}$. Age is a crucial factor that contributes to patients' overall health, prefracture mobility, and postoperative recovery rate $^{12}$. The primary causes of UTI include postoperative urinary retention and neurogenic bladder dysfunction ${ }^{13}$. UTIs are more common in women than in men because their urethras are relatively short and close to the anus and vagina. Escherichia coli is the most common pathogen implicated in UTIs ${ }^{14}$. These findings are consistent with those of other studies ${ }^{15-17}$.

Logistic regression analysis revealed that the comorbidities of HTN, DM, CAD, hyperlipidaemia, CRF, CVA, and depression were all significantly correlated with high S-UTI incidence. CRF and CVA were the most critical effect modifiers. CRF is associated with UTI for two main reasons. First, oliguria in CRF may cause stagnation along the urinary tract, thereby promoting bacterial growth. Second, defective urinary concentration affects the concentration of antibacterial substances in the urine ${ }^{18,19}$. In addition, acute urine retention and other micturition disorders, which occur frequently in elderly patients after hip fracture surgery, may aggravate UTIs to develop into S-UTIs ${ }^{13}$. UTIs affect between 10 and $19 \%$ of patients with CVA and are highly common poststroke. Comorbid CVA in elderly patients with hip fracture may increase the incidence of S-UTI ${ }^{20}$. According to Donegan et al., classes III and IV in the American Society of Anaesthesiologists classification of physiological status are strongly associated with perioperative complications, such as CVA and CRF, in elderly patients after hip fracture surgery ${ }^{21}$. This result is consistent with the present findings.

We divided the study group into ORIF and hemiarthroplasty groups for analysis in the present study. Each surgical method was indicated for hip fracture on the basis of fracture type and classification. According to a previous literature review, hemiarthroplasty is more cost effective than ORIF with screw fixation for nondisplaced femoral neck fractures in elderly patients with low function demand ${ }^{22}$. Another study revealed no differences in 30-day mortality rates but significant differences in respiratory complications among ORIF, hemiarthroplasty, and total hip arthroplasty groups ${ }^{23}$. The present study results reveal no significant difference in S-UTI incidence between the ORIF and hemiarthroplasty groups. Patient characteristics and comorbidities may be as crucial as fracture type is for the selection of an appropriate surgical method. We also excluded patients who underwent surgery more than $24 \mathrm{~h}$ after fracture because a previous meta-analysis of more than 190,000 patients indicated that delayed surgery for hip fracture is associated with a significantly increased risk of death and pressure sores ${ }^{24}$. In 2004, the Journal of the American Medical Association revealed that surgery within $24 \mathrm{~h}$ of fracture is associated with reduced pain and duration of hospitalisation; therefore, patients with hip fracture in stable medical condition should undergo surgery as soon as possible ${ }^{25}$. 


\begin{tabular}{|c|c|c|c|c|c|c|c|c|c|c|c|c|c|c|c|c|}
\hline \multirow[b]{3}{*}{ Code } & \multirow{2}{*}{\multicolumn{3}{|c|}{\begin{tabular}{|l|} 
Non-hip fx \\
0
\end{tabular}}} & \multirow{2}{*}{\multicolumn{3}{|c|}{\begin{tabular}{|l|} 
ORIF \\
1
\end{tabular}}} & \multirow{2}{*}{\multicolumn{3}{|c|}{\begin{tabular}{|l|} 
Hemiarthroplasty \\
2
\end{tabular}}} & \multicolumn{7}{|c|}{ Hazard ratio ( $95 \%$ confidence interval) } \\
\hline & & & & & & & & & & \multicolumn{3}{|l|}{ Crude } & \multicolumn{3}{|l|}{ Adjusted } & \multirow{2}{*}{$\begin{array}{l}P \text { for } \\
\text { interaction }\end{array}$} \\
\hline & Event & PY & IR & Event & PY & IR & Event & PY & IR & 1 vs 0 & 2 vs 0 & 2 vs 1 & 1 vs 0 & 2 vs 0 & 2 vs 1 & \\
\hline Overall & 5106 & 95,710 & 5.3 & 1071 & 12,927 & 8.3 & 756 & 8685 & 8.7 & $\begin{array}{l}1.54(1.45 \\
1.65)^{\star * \star *}\end{array}$ & $\begin{array}{l}1.62(1.5 \\
1.75)^{\star \star \star}\end{array}$ & $\begin{array}{l}1.04 \\
(0.95-1.15)\end{array}$ & $\begin{array}{l}1.65(1.55 \\
1.77)^{* * *}\end{array}$ & $\begin{array}{l}1.55(1.44 \\
1.68)^{* * *}\end{array}$ & \begin{tabular}{|l|}
0.93 \\
$(0.85-1.03)$
\end{tabular} & \\
\hline \multicolumn{17}{|l|}{ Sex } \\
\hline Female & 3139 & 52,931 & 5.9 & 629 & 6827 & 9.2 & 504 & 5634 & 8.9 & $\begin{array}{l}1.55(1.42, \\
1.68)^{* * *}\end{array}$ & $\begin{array}{l}1.5(1.37 \\
1.65)^{* * *}\end{array}$ & \begin{tabular}{|l|}
0.97 \\
$(0.86-1.09)$
\end{tabular} & $\begin{array}{l}1.51(1.38 \\
1.64)^{* * *}\end{array}$ & $\begin{array}{l}1.39(1.27 \\
1.53)^{* * *}\end{array}$ & \begin{tabular}{|l|}
0.92 \\
$(0.82-1.03)$
\end{tabular} & \\
\hline Male & 1967 & 42,779 & 4.6 & 442 & 6100 & 7.2 & 252 & 3051 & 8.3 & $\begin{array}{l}1.57(1.41 \\
1.74)^{\star * \star}\end{array}$ & $\begin{array}{l}1.78(1.56 \\
2.02)^{\star * \star}\end{array}$ & $\begin{array}{l}1.13 \\
(0.97-1.32)\end{array}$ & $\begin{array}{l}1.9(1.72, \\
2.11)^{\star * *}\end{array}$ & $\begin{array}{l}1.97(1.73 \\
2.25)^{* * \star}\end{array}$ & $\begin{array}{l}1.03 \\
(0.88-1.21)\end{array}$ & \\
\hline \multicolumn{17}{|c|}{ Age (years) } \\
\hline $50-59$ & 119 & 10,977 & 1.1 & 40 & 2164 & 1.8 & 12 & 315 & 3.8 & $\begin{array}{l}1.69(1.18 \\
2.42)^{* *}\end{array}$ & $\begin{array}{l}3.48(1.92, \\
6.3)^{\star * *}\end{array}$ & $\begin{array}{l}2.05(1.07- \\
3.91)^{*}\end{array}$ & $\begin{array}{l}1.72(1.19 \\
2.47)^{\star *}\end{array}$ & $\begin{array}{l}2.3(1.23 \\
4.3)^{* *}\end{array}$ & $\begin{array}{l}1.33 \\
(0.68-2.62)\end{array}$ & \\
\hline 60-69 & 485 & 18,865 & 2.6 & 101 & 2276 & 4.4 & 99 & 1957 & 5.1 & $\begin{array}{l}1.72(1.39 \\
2.13)^{* * *}\end{array}$ & $\begin{array}{l}1.96(1.58 \\
2.44)^{* * *}\end{array}$ & $\begin{array}{l}1.14 \\
(0.86-1.50)\end{array}$ & $\begin{array}{l}1.7(1.37 \\
2.11)^{* * *}\end{array}$ & $\begin{array}{l}1.76(1.42 \\
2.19)^{* * *}\end{array}$ & $\begin{array}{l}1.03 \\
(0.78-1.36)\end{array}$ & \\
\hline $70-79$ & 2002 & 37,630 & 5.3 & 430 & 4595 & 9.4 & 325 & 3917 & 8.3 & $\begin{array}{l}1.75(1.58 \\
1.94)^{* * *}\end{array}$ & $\begin{array}{l}1.55(1.38 \\
1.75)^{\star * *}\end{array}$ & \begin{tabular}{|l|}
0.88 \\
$(0.76-1.02)$
\end{tabular} & $\begin{array}{l}1.78(1.6 \\
1.97)^{\star * *}\end{array}$ & $\begin{array}{l}1.6(1.42 \\
1.8)^{* * *}\end{array}$ & \begin{tabular}{|l|}
0.90 \\
$(0.78-1.04)$
\end{tabular} & \\
\hline $80+$ & 2500 & 28,237 & 8.9 & 500 & 3891 & 13 & 320 & 2496 & 13 & $\begin{array}{l}1.45(1.31 \\
1.59)^{\star * *}\end{array}$ & $\begin{array}{l}1.44(1.28 \\
1.62)^{\star * *}\end{array}$ & $\begin{array}{l}0.99 \\
(0.86-1.14)\end{array}$ & $\begin{array}{l}1.5(1.36 \\
1.65)^{\star * *}\end{array}$ & $\begin{array}{l}1.39(1.24 \\
1.56)^{* * *}\end{array}$ & \begin{tabular}{|l|}
0.92 \\
$(0.80-1.06)$
\end{tabular} & \\
\hline \multicolumn{17}{|c|}{ Comorbidity } \\
\hline \multicolumn{17}{|l|}{ HTN } \\
\hline No & 1038 & 29,247 & 3.5 & 228 & 4548 & 5 & 142 & 2488 & 5.7 & $\begin{array}{l}1.41(1.22 \\
1.63)^{* * *}\end{array}$ & $\begin{array}{l}1.6(1.35 \\
1.91)^{\star * *}\end{array}$ & \begin{tabular}{|l|}
1.13 \\
$(0.92-1.40)$
\end{tabular} & $\begin{array}{l}1.53(1.32 \\
1.76)^{* * *}\end{array}$ & $\begin{array}{l}1.6(1.34 \\
1.91)^{* * *}\end{array}$ & \begin{tabular}{|l|}
1.05 \\
$(0.85-1.29)$
\end{tabular} & \\
\hline Yes & 4068 & 66,462 & 6.1 & 843 & 8379 & 10 & 614 & 6197 & 9.9 & $\begin{array}{l}1.63(1.51, \\
1.76)^{\star * \star}\end{array}$ & $\begin{array}{l}1.61(1.48, \\
1.75)^{\star * \star}\end{array}$ & \begin{tabular}{|l|}
0.98 \\
$(0.88-1.09)$ \\
\end{tabular} & $\begin{array}{l}1.69(1.57 \\
1.82)^{* * *}\end{array}$ & $\begin{array}{l}1.55(1.42 \\
1.69)^{* * *}\end{array}$ & \begin{tabular}{|l|}
0.91 \\
$(0.82-1.01)$ \\
\end{tabular} & 0.210 \\
\hline \multicolumn{17}{|l|}{$\mathrm{DM}$} \\
\hline No & 2731 & 60,377 & 4.5 & 603 & 8372 & 7.2 & 407 & 5526 & 7.4 & $\begin{array}{l}1.59(1.45 \\
1.73)^{\star * \star} \\
\end{array}$ & $\begin{array}{l}1.62(1.46 \\
1.8)^{\star * \star}\end{array}$ & \begin{tabular}{|l|}
1.02 \\
$(0.90-1.15)$ \\
\end{tabular} & $\begin{array}{l}1.66(1.52 \\
1.82)^{* * \star} \\
\end{array}$ & \begin{tabular}{|l|}
$1.5(1.35$ \\
$1.66)^{* * *}$ \\
\end{tabular} & \begin{tabular}{|l|}
0.90 \\
$(0.79-1.02)$ \\
\end{tabular} & \\
\hline Yes & 2375 & 35,332 & 6.7 & 468 & 4555 & 10 & 349 & 3159 & 11 & $\begin{array}{l}1.51(1.37 \\
1.67)^{* * *}\end{array}$ & $\begin{array}{l}1.63(1.46 \\
1.82)^{\star * *}\end{array}$ & \begin{tabular}{|l|}
1.07 \\
$(0.93-1.23)$
\end{tabular} & $\begin{array}{l}1.62(1.47, \\
1.79)^{* * *}\end{array}$ & $\begin{array}{l}1.61(1.44, \\
1.8)^{* * *}\end{array}$ & \begin{tabular}{|l|}
0.99 \\
$(0.86-1.13)$
\end{tabular} & 0.764 \\
\hline \multicolumn{17}{|c|}{ Hyperlipidemia } \\
\hline No & 3627 & 66,219 & 5.5 & 770 & 9295 & 8.3 & 510 & 5912 & 8.6 & $\begin{array}{l}1.51(1.39 \\
1.63)^{\star * *}\end{array}$ & $\begin{array}{l}1.57(1.43 \\
1.72)^{\star * \star}\end{array}$ & \begin{tabular}{|l|}
1.04 \\
$(0.93-1.16)$
\end{tabular} & $\begin{array}{l}1.61(1.49, \\
1.74)^{* * *}\end{array}$ & $\begin{array}{l}1.52(1.38 \\
1.66)^{* * *}\end{array}$ & \begin{tabular}{|l|}
0.94 \\
$(0.84-1.05)$
\end{tabular} & \\
\hline Yes & 1479 & 29,491 & 5 & 301 & 3632 & 8.3 & 246 & 2773 & 8.9 & $\begin{array}{l}1.65(1.45 \\
1.86)^{\star * \star *}\end{array}$ & $\begin{array}{l}1.75(1.53 \\
2.01)^{\star * \star}\end{array}$ & $\begin{array}{l}1.06 \\
(0.90-1.26)\end{array}$ & $\begin{array}{l}1.74(1.54, \\
1.97)^{* * *}\end{array}$ & $\begin{array}{l}1.65(1.44 \\
1.89)^{* * \star}\end{array}$ & \begin{tabular}{|l|}
0.94 \\
$(0.79-1.11)$
\end{tabular} & 0.291 \\
\hline \multicolumn{17}{|l|}{ CRF } \\
\hline No & 4650 & 94,193 & 4.9 & 1008 & 12,776 & 7.9 & 707 & 8532 & 8.3 & $\begin{array}{l}1.59(1.49 \\
1.7)^{\star * *}\end{array}$ & $\begin{array}{l}1.67(1.54 \\
1.8)^{* * *}\end{array}$ & \begin{tabular}{|l|}
1.04 \\
$(0.95-1.15)$
\end{tabular} & $\begin{array}{l}1.66(1.55 \\
1.78)^{* * *}\end{array}$ & $\begin{array}{l}1.58(1.46 \\
1.72)^{* * *}\end{array}$ & \begin{tabular}{|l|}
0.95 \\
$(0.86-1.05)$
\end{tabular} & \\
\hline Yes & 456 & 1517 & 30 & 63 & 151 & 42 & 49 & 153 & 32 & $\begin{array}{l}1.45(1.11 \\
1.89)^{\star *}\end{array}$ & $\begin{array}{l}1.11(0.82 \\
1.49)\end{array}$ & $\begin{array}{l}0.76 \\
(0.52-1.10)\end{array}$ & $\begin{array}{l}1.56(1.19 \\
2.05)^{\star *}\end{array}$ & $\begin{array}{l}1.2(0.88 \\
1.63)\end{array}$ & $\begin{array}{l}0.76 \\
(0.52-1.12)\end{array}$ & $0.0097^{* *}$ \\
\hline \multicolumn{17}{|c|}{ Depression } \\
\hline No & 4454 & 85,443 & 5.2 & 943 & 11,583 & 8.1 & 654 & 7635 & 8.6 & $\begin{array}{l}1.55(1.45 \\
1.67)^{* * *}\end{array}$ & $\begin{array}{l}1.63(1.5 \\
1.77)^{\star \star \star}\end{array}$ & \begin{tabular}{|l|}
1.05 \\
$(0.95-1.16)$
\end{tabular} & $\begin{array}{l}.67(1.56 \\
1.79)^{* * *}\end{array}$ & $\begin{array}{l}1.56(1.44 \\
1.69)^{* * *}\end{array}$ & \begin{tabular}{|l|}
0.93 \\
$(0.55-0.64)$
\end{tabular} & \\
\hline Yes & 652 & 10,267 & 6.4 & 128 & 1344 & 9.5 & 102 & 1049 & 9.7 & $\begin{array}{l}1.49(1.24 \\
1.81)^{* * *}\end{array}$ & $\begin{array}{l}1.52(1.23 \\
1.87)^{\star * *}\end{array}$ & \begin{tabular}{|l|}
1.01 \\
$(0.78-1.31)$
\end{tabular} & $\begin{array}{l}1.53(1.27 \\
1.85)^{* * *}\end{array}$ & $\begin{array}{l}1.51(1.22 \\
1.86)^{* * *}\end{array}$ & \begin{tabular}{|l|}
0.98 \\
$(0.75-1.27)$
\end{tabular} & 0.7879 \\
\hline \multicolumn{17}{|l|}{ CAD } \\
\hline No & 2342 & 54,039 & 4.3 & 534 & 7722 & 6.9 & 370 & 4781 & 7.7 & $\begin{array}{l}1.59(1.45 \\
1.75)^{\star \star \star *}\end{array}$ & $\begin{array}{l}.78(1.59 \\
1.98)^{\star * \star}\end{array}$ & \begin{tabular}{|l|}
1.11 \\
$(0.97-1.27)$
\end{tabular} & $\begin{array}{l}1.74(1.58 \\
1.91)^{* * *}\end{array}$ & $\begin{array}{l}1.6(1.43 \\
1.79)^{* * *}\end{array}$ & \begin{tabular}{|l|}
0.91 \\
$(0.80-1.05)$
\end{tabular} & \\
\hline Yes & 2764 & 41,671 & 6.6 & 537 & 5205 & 10 & 386 & 3904 & 9.9 & $\begin{array}{l}1.54(1.41, \\
1.69)^{* \star \star}\end{array}$ & $\begin{array}{l}1.48(1.33 \\
1.65)^{\star * \star}\end{array}$ & \begin{tabular}{|l|}
0.96 \\
$(0.84-1.09)$
\end{tabular} & $\begin{array}{l}1.57(1.43 \\
1.72)^{* * *}\end{array}$ & $\begin{array}{l}1.51(1.35 \\
1.68)^{* * *}\end{array}$ & \begin{tabular}{|l|}
0.96 \\
$(0.84-1.09)$
\end{tabular} & 0.0648 \\
\hline \multicolumn{17}{|l|}{ CVA } \\
\hline No & 2608 & 64,539 & 4 & 597 & 9000 & 6.6 & 405 & 5554 & 7.3 & $\begin{array}{l}1.64(1.5 \\
1.79)^{\star * *} \\
\end{array}$ & $\begin{array}{l}1.8(1.62 \\
1.99)^{* * *}\end{array}$ & \begin{tabular}{|l|}
1.09 \\
$(0.96-1.24)$
\end{tabular} & $\begin{array}{l}1.7(1.55 \\
1.86)^{* * *}\end{array}$ & $\begin{array}{l}1.63(1.47 \\
1.82)^{* * *}\end{array}$ & \begin{tabular}{|l|}
0.96 \\
$(0.84-1.09)$
\end{tabular} & \\
\hline Yes & 2498 & 31,170 & 8 & 474 & 3927 & 12 & 351 & 3130 & 11 & $\begin{array}{l}1.49(1.35 \\
1.65)^{\star * *}\end{array}$ & $\begin{array}{l}1.39(1.25 \\
1.56)^{\star * *}\end{array}$ & $\begin{array}{l}0.93 \\
(0.81-1.07)\end{array}$ & $\begin{array}{l}1.58(1.44 \\
1.75)^{* * *}\end{array}$ & $\begin{array}{l}1.46(1.31 \\
1.63)^{* * *}\end{array}$ & \begin{tabular}{|l|}
0.92 \\
$(0.80-1.05)$
\end{tabular} & $0.0038^{* *}$ \\
\hline
\end{tabular}

Table 2. Incidences and hazard ratios of S-UTI for ORIF, hemiarthroplasty, and non-hip fx cohorts. Adjusted hazard ratio for controlling for sex, age, and every comorbidity in Table 3. HR hazard ratio, IR incidence rate, per 100 person-years, $C A D$ coronary artery disease, $C I$ confidence interval, $C R F$ chronic renal failure, $C V A$ cerebrovascular accident, $D M$ diabetes mellitus, $f x$ fracture, HTN hypertension, ORIF open reduction and internal fixation, $P Y$ person-years, $S$ - $U T I$ severe urinary tract infection. ${ }^{*} P<0.05,{ }^{*} P<0.01,{ }^{* *} P<0.001$.

This study did not include patients who did not undergo surgery for hip fracture because surgery is the primary intervention for this type of injury ${ }^{25}$. The large sample size was a strength of this study; the comprehensive 


\begin{tabular}{|c|c|c|c|c|c|c|c|}
\hline \multirow[b]{2}{*}{ Characteristics } & \multirow[b]{2}{*}{ S-UTI event no. $(n=6933)$} & \multicolumn{3}{|c|}{ Crude } & \multicolumn{3}{|c|}{ Adjusted } \\
\hline & & HR & $(95 \% \mathrm{CI})$ & $P$ value & HR & $(95 \% \mathrm{CI})$ & $P$ value \\
\hline \multicolumn{8}{|l|}{ Hip fx } \\
\hline No & 5106 & 1 & Reference & & 1 & Reference & \\
\hline ORIF & 1071 & 1.54 & $(1.45,1.65)$ & $<0.001$ & 1.65 & $(1.55,1.77)$ & $<0.001$ \\
\hline Hemiarthroplasty & 756 & 1.62 & $(1.5,1.75)$ & $<0.001$ & 1.55 & $(1.44,1.68)$ & $<0.001$ \\
\hline \multicolumn{8}{|l|}{ Sex } \\
\hline Female & 4272 & 1 & Reference & & 1 & Reference & \\
\hline Male & 2661 & 0.78 & $(0.74,0.82)$ & $<0.001$ & 0.77 & $(0.74,0.81)$ & $<0.001$ \\
\hline \multicolumn{8}{|l|}{ Age (years) } \\
\hline $50-59$ & 171 & 1 & Reference & & 1 & Reference & \\
\hline $60-69$ & 685 & 2.32 & $(1.97,2.75)$ & $<0.001$ & 1.87 & $(1.58,2.22)$ & $<0.001$ \\
\hline $70-79$ & 2757 & 4.64 & $(3.98,5.42)$ & $<0.001$ & 3.42 & $(2.93,4.01)$ & $<0.001$ \\
\hline $80+$ & 3320 & 7.3 & $(6.26,8.52)$ & $<0.001$ & 5.57 & $(4.76,6.52)$ & $<0.001$ \\
\hline \multicolumn{8}{|l|}{ Comorbidity } \\
\hline \multicolumn{8}{|l|}{ HTN } \\
\hline No & 1408 & 1 & Reference & & 1 & Reference & \\
\hline Yes & 5525 & 1.71 & $(1.61,1.82)$ & $<0.001$ & 1.2 & $(1.12,1.28)$ & $<0.001$ \\
\hline \multicolumn{8}{|l|}{$\mathrm{DM}$} \\
\hline No & 3741 & 1 & Reference & & 1 & Reference & \\
\hline Yes & 3192 & 1.45 & $(1.38,1.52)$ & $<0.001$ & 1.46 & $(1.38,1.53)$ & $<.001$ \\
\hline \multicolumn{8}{|l|}{ Hyperlipidemia } \\
\hline No & 4907 & 1 & Reference & & 1 & Reference & \\
\hline Yes & 2026 & 0.92 & $(0.88,0.97)$ & 0.002 & 0.74 & $(0.7,0.78)$ & $<0.001$ \\
\hline \multicolumn{8}{|l|}{ CRF } \\
\hline No & 6365 & 1 & Reference & & 1 & Reference & \\
\hline Yes & 568 & 5.51 & $(5.06,6)$ & $<0.001$ & 4.85 & $(4.45,5.29)$ & $<0.001$ \\
\hline \multicolumn{8}{|l|}{ Depression } \\
\hline No & 6051 & 1 & Reference & & 1 & Reference & \\
\hline Yes & 882 & 1.18 & $(1.1,1.27)$ & $<0.001$ & 1.1 & $(1.02,1.18)$ & 0.01 \\
\hline \multicolumn{8}{|l|}{ CAD } \\
\hline No & 3246 & 1 & Reference & & 1 & Reference & \\
\hline Yes & 3687 & 1.46 & $(1.4,1.53)$ & $<0.001$ & 1.06 & $(1.01,1.12)$ & 0.02 \\
\hline \multicolumn{8}{|l|}{ CVA } \\
\hline No & 3610 & 1 & Reference & & 1 & Reference & \\
\hline Yes & 3323 & 1.86 & $(1.77,1.95)$ & $<0.001$ & 1.56 & $(1.48,1.64)$ & $<0.001$ \\
\hline
\end{tabular}

Table 3. Cox model measured hazard ratio and 95\% confidence intervals of S-UTI associated with ORIF, hemiarthroplasty, non-hip fx cohorts and covariates. Adjusted HR: adjusted for femoral neck fracture, sex, age, and every comorbidity in Table 2 in Cox proportional hazards regression. CAD coronary artery disease, $C I$ confidence interval, $C R F$ chronic renal failure, $C V A$ cerebrovascular accident, $D M$ diabetes mellitus, $f x$ fracture, $H R$ hazard ratio, HTN hypertension, ORIF open reduction and internal fixation, $S$-UTI severe urinary tract infection.

coverage of the National Health Insurance (NHI) system (covering $>95 \%$ of the Taiwanese population) may have minimised selection and nonresponse biases. However, this study had some limitations. First, data on lifestyle factors, detailed disease symptoms, personal characteristics, and biochemical indices, which may be influential sources of bias, were unavailable because the National Health Insurance Research Database (NHIRD) does not provide this information. Second, because our results are based on data from Taiwan, the present findings may not be directly generalisable to Caucasian or African populations. Despite these limitations, the present study indicated the importance of preventing S-UTI after surgical intervention for hip fracture in elderly patients. Clinicians and family members alike should collaborate to prevent S-UTI and thus improve the quality of life of patients during and after recovery from hip fracture.

\section{Conclusions}

The S-UTI incidence in the study cohort was higher than that in the comparison cohort, particularly in patients who were older, female, or had comorbid CVA or CRF. To increase patients' postoperative quality of life, further prevention or protection protocols for these patients at high risk of S-UTI should be enacted earlier during the recovery period after hip fracture. 


\begin{tabular}{|c|c|c|c|c|}
\hline Variables & $n$ & Event no & Adjusted HR (95\% CI) & $P$ value $^{\mathrm{a}}$ \\
\hline \multicolumn{5}{|c|}{ Hip fx status } \\
\hline CRF & & & & $0.010^{*}$ \\
\hline \multicolumn{5}{|l|}{ No } \\
\hline No & 22,639 & 4650 & 1 (Reference) & \\
\hline Yes & 457 & 456 & $5.05(4.58-5.56)$ & $<0.0001$ \\
\hline \multicolumn{5}{|l|}{ ORIF } \\
\hline No & 3405 & 1008 & $1.66(1.55-1.78)$ & $<0.0001$ \\
\hline Yes & 64 & 63 & $7.77(6.06-9.97)$ & $<0.0001$ \\
\hline \multicolumn{5}{|c|}{ Hemiarthroplasty } \\
\hline No & 2256 & 707 & $1.59(1.46-1.72)$ & $<0.0001$ \\
\hline Yes & 49 & 49 & $5.85(4.41-7.76)$ & $<0.0001$ \\
\hline \multicolumn{5}{|c|}{ Hip fx status } \\
\hline CVA & & & & $0.004^{*}$ \\
\hline \multicolumn{5}{|l|}{ No } \\
\hline No & 14,304 & 2608 & 1 (Reference) & \\
\hline Yes & 8792 & 2498 & $1.58(1.50-1.68)$ & $<0.0001$ \\
\hline \multicolumn{5}{|l|}{ ORIF } \\
\hline No & 2208 & 597 & $1.69(1.54-1.85)$ & $<0.0001$ \\
\hline Yes & 1261 & 474 & $2.55(2.31-2.81)$ & $<0.0001$ \\
\hline \multicolumn{5}{|c|}{ Hemiarthroplasty } \\
\hline No & 1373 & 405 & $1.64(1.47-1.82)$ & $<0.0001$ \\
\hline Yes & 932 & 351 & $2.32(2.07-2.60)$ & $<0.0001$ \\
\hline
\end{tabular}

Table 4. Cox proportional hazard regression analysis for the risk of hip fx-associated S-UTI with interaction of CRF and CVA. Adjusted HR: adjusted for femoral neck fracture, sex, age, and the comorbidities in Cox proportional hazards regression. $H R$ hazard ratio, $C I$ confidence interval, $C R F$ chronic renal failure, $C V A$ cerebrovascular accident, $f x$ fracture, ORIF open reduction and internal fixation, $S$-UTI severe urinary tract infection. ${ }^{\mathrm{a} P}$ value for interaction.

\section{Methods}

The main data source for this study was the NHIRD, access to which is provided by the National Health Research Institute. The NHIRD is a nationwide database covering approximately $99 \%$ of the 23.74 million residents of Taiwan who are enrolled in the NHI programme, which was launched on March 1, 1995. We used scrambled identification to link three additional data sources, namely the Registry for Catastrophic Illness Patient Database (RCIPD), Longitudinal Health Insurance Database 2000 (LHID 2000), and Registry of Beneficiaries (RB) ${ }^{26}$. Records in the NHIRD correspond with the codes of the International Classification of Diseases, Ninth Revision, Clinical Modification (ICD-9-CM). This study was approved by the Ethics Review Board of China Medical University and China Medical University Hospital (CMUH-104-REC2-115), and all analyses were performed in accordance with relevant guidelines and regulations. The RCIPD, LHID 2000, and RB, as legal and delinked databases for research, are anonymised and maintained by the National Health Research Institute with confidentiality in accordance with the Personal Electronic Data Protection Law. The requirement for informed consent of this study was waived by the Ethics Review Board of China Medical University and China Medical University Hospital on the basis of the Personal Electronic Data Protection Law.

We searched the RCIPD from 1 January 2000 to 31 December 2012 for patients aged $\geq 50$ years receiving surgical intervention for newly diagnosed hip fracture (ICD-9-CM codes 820.0-820.9; codes for ORIF and hemiarthroplasty: 64029B and 64170B). These patients constituted the study cohort. S-UTI was defined as UTI requiring hospitalisation (ICD-9-CM code 599.0). The comparison cohort comprised patients from the LHID 2000 without hip fracture during the same period. In both cohorts, patients with any history of S-UTI before or during the admission period for hip fracture surgery, those with a confirmed diagnosis of hospital-acquired UTI from prolonged Foley catheter placement, and those receiving antibiotics more than $24 \mathrm{~h}$ postoperatively before and during the admission period for hip fracture surgery were excluded. In the hip fracture cohort, patients who did not undergo surgery within $72 \mathrm{~h}$ after fracture were excluded. The study and comparison cohorts were frequency matched for age, sex, index year, and comorbidities at a 1:4 ratio.

All individuals were followed from the index date to hospitalisation for S-UTI until their date of death, withdrawal from the NHI programme, or 31 December 2012, whichever occurred first. Some demographic factors and comorbidities that may be associated with S-UTI were also identified. These included sexes, age, and the following comorbidities: HTN (ICD-9-CM codes 401-405), DM (ICD-9-CM code 250), hyperlipidaemia (ICD-9-CM code 272), depression (ICD-9-CM codes 296.2, 296.3, 296.82, 300.4, 309.0, 309.1, and 311), CAD (ICD-9 codes 410-414), CVA (ICD-9 codes 430-438), and CRF (ICD-9 code 585). 
Statistical analysis. The standardised mean differences in sex, age, comorbidities, and follow-up duration were subjected to further analysis. The incidence rate was defined as the number of S-UTI events divided by person-years. Crude hazard ratios, aHRs, and 95\% CIs were calculated using the multivariable Cox proportional hazard regression model (adjusted for sex, age, and comorbidities). The Kaplan-Meier method was used to determine the cumulative incidence of S-UTI in patients with and without hip fracture, and the log-rank test was used to examine its significance. The analyses were performed using SAS software, Version 9.4 of the SAS System for Unix (SAS Institute, Cary, NC, USA). A $P$ value of $<0.05$ was considered to be significant.

Ethics approval and consent to participate. This study was approved by the Ethics Review Board of China Medical University and China Medical University Hospital (CMUH-104-REC2-115) and confirmed that all the experiments were performed in accordance with relevant guidelines and regulations. The requirement for informed consent of this study was waived by the Ethics Review Board based on the Personal Electronic Data Protection Law because the NHIRD database are anonymized and maintained by the National Health Research Institute with confidentiality according to the law.

\section{Data availability}

The datasets used and analyzed during the current study are available from the corresponding author on reasonable request.

Received: 26 October 2020; Accepted: 21 January 2021

Published online: 09 February 2021

\section{References}

1. Kanis, J. A. et al. A systematic review of hip fracture incidence and probability of fracture worldwide. Osteoporos. Int. 23, $2239-2256$. https://doi.org/10.1007/s00198-012-1964-3 (2012).

2. Simunovic, N. et al. Effect of early surgery after hip fracture on mortality and complications: Systematic review and meta-analysis. CMAJ 182, 1609-1616. https://doi.org/10.1503/cmaj.092220 (2010).

3. Bliemel, C. et al. Impact of Parkinson's disease on the acute care treatment and medium-term functional outcome in geriatric hip fracture patients. Arch. Orthop. Trauma Surg. 135, 1519-1526. https://doi.org/10.1007/s00402-015-2298-3 (2015).

4. Nurmi, I., Narinen, A., Lüthje, P. \& Tanninen, S. Functional outcome and survival after hip fracture in elderly: A prospective study of 106 consecutive patients. J. Orthop. Traumatol. 5, 7-14. https://doi.org/10.1007/s10195-004-0033-x (2004).

5. Bhandari, M. et al. Internal fixation compared with arthroplasty for displaced fractures of the femoral neck: A meta-analysis. J. Bone Joint Surg. Am. 85, 1673-1681. https://doi.org/10.2106/00004623-200309000-00004 (2003).

6. LeBlanc, E. S. et al. Hip fracture and increased short-term but not long-term mortality in healthy older women. Arch. Intern. Med. 171, 1831-1837. https://doi.org/10.1001/archinternmed.2011.447 (2011).

7. McCoy, C. C. et al. Impact of specific postoperative complications on the outcomes of emergency general surgery patients. J. Trauma Acute Care Surg. 78, 912-918. https://doi.org/10.1097/TA.0000000000000611 (2015).

8. Bliemel, C. et al. Urinary tract infection in patients with hip fracture: An underestimated event?. Geriatr. Gerontol. Int. 17, 23692375. https://doi.org/10.1111/ggi.13077 (2017).

9. Tan, S. T., Tan, W. P., Jaipaul, J., Chan, S. P. \& Sathappan, S. S. Clinical outcomes and hospital length of stay in 2756 elderly patients with hip fractures: A comparison of surgical and non-surgical management. Singapore Med. J. 58, 253-257. https://doi.org/10.11622 /smedj.2016045 (2017).

10. Bouvet, C. et al. Is there any benefit in pre-operative urinary analysis before elective total joint replacement?. Bone Joint J. 96-B, 390-394. https://doi.org/10.1302/0301-620X.96B3.32620 (2014).

11. Kamel, H. K. The frequency and factors linked to a urinary tract infection coding in patients undergoing hip fracture surgery. J. Am. Med. Dir. Assoc. 6, 316-320. https://doi.org/10.1016/j.jamda.2005.04.005 (2005).

12. Shah, M. R., Aharonoff, G. B., Wolinsky, P., Zuckerman, J. D. \& Koval, K. J. Outcome after hip fracture in individuals ninety years of age and older. J. Orthop. Trauma 15, 34-39. https://doi.org/10.1097/00005131-200101000-00007 (2001).

13. Johansson, R. M. \& Christensson, L. Urinary retention in older patients in connection with hip fracture surgery. J. Clin. Nurs. 19, 2110-2116. https://doi.org/10.1111/j.1365-2702.2010.03261.x (2010).

14. Valiquette, L. Urinary tract infections in women. Can. J. Urol. 8(Suppl 1), 6-12 (2001).

15. Hedström, M., Gröndal, L. \& Ahl, T. Urinary tract infection in patients with hip fractures. Injury 30, 341-343. https://doi. org/10.1016/s0020-1383(99)00094-7 (1999).

16. Nyman, M. H., Johansson, J. E., Persson, K. \& Gustafsson, M. A prospective study of nosocomial urinary tract infection in hip fracture patients. J. Clin. Nurs. 20, 2531-2539. https://doi.org/10.1111/j.1365-2702.2011.03769.x (2011).

17. Nyman, M. H. et al. Intermittent versus indwelling urinary catheterisation in hip surgery patients: A randomised controlled trial with cost-effectiveness analysis. Int. J. Nurs. Stud. 50, 1589-1598. https://doi.org/10.1016/j.ijnurstu.2013.05.007 (2013).

18. Saitoh, H., Nakamura, K., Hida, M. \& Satoh, T. Urinary tract infection in oliguric patients with chronic renal failure. J. Urol. 133, 990-993. https://doi.org/10.1016/s0022-5347(17)49344-x (1985).

19. Hsiao, C. Y. et al. Urinary tract infection in patients with chronic kidney disease. Turk. J. Med. Sci. 44, 145-149. https://doi. org/10.3906/sag-1303-51 (2014).

20. Smith, C., Almallouhi, E. \& Feng, W. Urinary tract infection after stroke: A narrative review. J. Neurol. Sci. 403, 146-152. https:// doi.org/10.1016/j.jns.2019.06.005 (2019).

21. Donegan, D. J. et al. Use of medical comorbidities to predict complications after hip fracture surgery in the elderly. J. Bone Joint Surg. Am. 92, 807-813. https://doi.org/10.2106/JBJS.I.00571 (2010).

22. Yong, T. M. et al. Screw fixation versus hemiarthroplasty for nondisplaced femoral neck fractures in the elderly: A cost-effectiveness analysis. J. Orthop. Trauma 34(7), 348-355. https://doi.org/10.1097/BOT.0000000000001747 (2020).

23. Fisher, M. A. et al. Open reduction internal fixation versus hemiarthroplasty versus total hip arthroplasty in the elderly: A review of the National Surgical Quality Improvement Program database. J Surg Res. 181(2), 193-198. https://doi.org/10.1016/j.jss.2012.07.004 (2013) (Epub 2012 Jul 20 PMID: 23122668).

24. Moja, L. et al. Timing matters in hip fracture surgery: Patients operated within 48 hours have better outcomes. A meta-analysis and meta-regression of over 190,000 patients. PLOS ONE 7(10), e46175. https://doi.org/10.1371/journal.pone.0046175 (2012) (Epub 2012 Oct 3. PMID: 23056256; PMCID: PMC3463569).

25. Orosz, G. M. et al. Association of timing of surgery for hip fracture and patient outcomes. JAMA 291(14), 1738-1743. https://doi. org/10.1001/jama.291.14.1738 (2004). 
26. Wu, T. Y., Majeed, A. \& Kuo, K. N. An overview of the healthcare system in Taiwan. Lond. J. Prim. Care (Abingdon) 3, 115-119. https://doi.org/10.1080/17571472.2010.11493315 (2010).

\section{Acknowledgements}

This manuscript was edited by Wallace Academic Editing.

\section{Author contributions}

K.-T.Y., T.-C.Y. and I.-H.C. conception and design of study. J.-H.W., K.-T.Y., H.-W.C. and C.-Y.H. acquisition of data. J.-H.W., K.-L.L. and C.-H.P. analysis and/or interpretation of data. Y.-C.L., Y.-C.H. and K.-T.Y. drafting the manuscript. W.-T.W. and R.-P.L. revising the manuscript critically for important intellectual content. All authors read and approved the final manuscript.

\section{Funding}

Taiwan Ministry of Health and Welfare Clinical Trial Center (MOHW108-TDU-B-212-133004), China Medical University Hospital, Academia Sinica Stroke Biosignature Project (BM10701010021), MOST Clinical Trial Consortium for Stroke (MOST 107-2321-B-039-004), Tseng-Lien Lin Foundation, Taichung, Taiwan, and Katsuzo and Kiyo Aoshima Memorial Funds, Japan.

\section{Competing interests}

The authors declare no competing interests.

\section{Additional information}

Correspondence and requests for materials should be addressed to K.-T.Y.

Reprints and permissions information is available at www.nature.com/reprints.

Publisher's note Springer Nature remains neutral with regard to jurisdictional claims in published maps and institutional affiliations.

(c) Open Access This article is licensed under a Creative Commons Attribution 4.0 International License, which permits use, sharing, adaptation, distribution and reproduction in any medium or format, as long as you give appropriate credit to the original author(s) and the source, provide a link to the Creative Commons licence, and indicate if changes were made. The images or other third party material in this article are included in the article's Creative Commons licence, unless indicated otherwise in a credit line to the material. If material is not included in the article's Creative Commons licence and your intended use is not permitted by statutory regulation or exceeds the permitted use, you will need to obtain permission directly from the copyright holder. To view a copy of this licence, visit http://creativecommons.org/licenses/by/4.0/.

(C) The Author(s) 2021 